\title{
Correction
}

\section{Correction: Khemka et al., "Dissecting the Function of Hippocampal Oscillations in a Human Anxiety Model”}

In the article "Dissecting the Function of Hippocampal Oscillations in a human Anxiety Model" by Saurabh Khemka, Gareth Barnes, Raymond J. Dolan, and Dominik R. Bach, which appeared on pages 6869-6876 of the July 19, 2017 issue, the Results section referred to a panel $C$ of Figure 4, which does not exist. The bold-typed reference link to Figure $4 C$ listed on page 6874, first column, last paragraph, line 7 should be removed from the sentence "To analyze this modulation, we averaged gamma $(30-80 \mathrm{~Hz})$ power for each point in time, and analyzed spectral modulation $(1-30 \mathrm{~Hz})$ of this gamma power envelope for each trial." This error does not affect any of the conclusions or interpretations in our paper. Mention of Figure $4 C$ has been removed from the online PDF version.

DOI:10.1523/JNEUROSCI.0161-18.2018 